\title{
A Novel Channel-Adaptive Uplink Access Control Protocol for Nomadic Computing
}

\author{
Yu-Kwong Kwok and Vincent K. N. Lau \\ Email: $\{y k w o k, k n l a u\} @ e e e . h k u . h k$ \\ Department of Electrical and Electronic Engineering \\ The University of Hong Kong, Pokfulam Road, Hong Kong \\ Tel: +852-2859-8059, Fax: +852-2559-8738 \\ Corresponding Author: Yu-Kwong Kwok
}

\begin{abstract}
We consider the uplink access control problem in a mobile computing system, which is based on a cellular phone network in that a user can use the mobile device to transmit voice or flle data. This resource management problem is important because efficient solution to uplink access control is critical for supporting a large user population with a reasonable level of quality of service (QoS). While there are a number of recently proposed protocols for uplink access control, these protocols possess a common drawback in that they do not exploit well the burst error properties, which are inevitable in a wireless communication system. In this paper, we propose a novel TDMA-based uplink access protocol, which employs a channel state dependent allocation strategy. Our protocol is motivated by two observations: (1) when channel state is bad, the throughput is low due to large amount of FEC (forward error correction) or excessive ARQ (automatic repeated request) is needed; and (2) because of (1), much of the mobile device's energy is wasted. The proposed protocol works closely with the underlying physical layer in that through observing the channel state information (CSI) of each mobile user, the MAC protocol first segregates a set of users with good CSI from requests gathered in the request contention phase of an uplink frame. The protocol then judiciously allocates channel bandwidth to contending users based on their channel conditions. Simulation results indicate that the proposed protocol considerably outperforms five state-of-the-art protocols in terms of packet loss, delay, and throughput.
\end{abstract}

Keywords: Mobile computing, distributed data access, wireless systems, adaptive protocols, error control.

\section{INTRODUCTION}

Tetherless nomadic computing environments are envisioned to proliferate in the near future due to the unprecedented advancements in hardware and wireless communication technologies. The use of wireless communication links allows users to continue computing while on the move. However, to realize a truly user-friendly mobile computing system entails tackling a number of intricate resource management and coordination chores such as efficient and reliable data dissemination, maintenance of data consistency, queries management, etc. [3]. The difficulties encountered in handling these tasks are aggravated by the fact that they have to be dealt with under a whole new set of system constraints like low data rates, high error rates, minimal power supply in the mobiles, etc. [5], [15]. Indeed, as it is widely believed that future mobile computing networks will be based on the ubiquitous cellular phone networks such that users are expected to use the a cell-phone like device for data transmission as well, resource management schemes must take into account the burst error characteristics and low power in the user's hand-held devices. While the problems involved in downlink data broadcasting are extensively studied by numerous researchers [4], $[11],[12],[13]$, the uplink access control problem has received much less attention. Efficient solution to uplink access control is critical for supporting a large user population with a reasonable level of quality of service $(Q \circ S)$. The abundant bandwidth available in the forthcoming third generation (3G) wireless systems does not help to alleviate the problem because the user population size will for sure increase proportionately. In this paper, we propose a novel TDMAbased uplink access protocol which judiciously allocates channel bandwidth to contending users based on their channel conditions. Our channel state dependent allocation is motivated by two observations: (1) when channel state is bad, the throughput is low due to large amount of FEC (forward error correction) or excessive ARQ (automatic repeated request) is needed; and (2) because of (1), much of the mobile device's energy will be wasted.

The classical design of an uplink access protocol is to arbitrate and statistically multiplex the transmission requests of multiple uncoordinated users and allocate transmission bandwidth to the users in a fair manner. Notorious examples include the ALOHA protocol for a packet radio network and the CSMA/CD protocol for a wired local area network. The key feature of the classic design is that all users are homogeneous - they have the same traffic characteristics. However, in our study we consider a cellular mobile information system for integrated voice 
(i.e., isochronous traffic) and file data services ${ }^{1}$ (i.e., bursty traffic), for which an effective and intelligent protocol is particularly desired due to the sharing of the precious bandwidth by a dynamically changing population of users with various traffic demands. There are four aspects in characterizing an uplink access control protocol:

- Request Mechanism: The mechanism of receiving user requests critically affects the performance of a protocol. For example, in some contention based protocols, too much contention (e.g., a large number of active users) will result in system instability such that users keep on contending without success due to excessive collisions. Under such a thrashing situation, most of the information slots are not used. Different protocols employ various techniques to combat this problem.

- Slots Allocation: In most protocols, information slots are assigned on a first-come-first-basis and can be reserved in subsequent frames if the user submits a voice request. However, some recently proposed protocols employ more intelligent approaches to further enhance the channel utilization.

- Frame Structure: Traditionally, the frame is of a static structure. That is, for example, there is a fixed portion of the frame dedicated for receiving transmission requests, while the remaining portion is for information slots. A major merit of a static frame structure is the ease of implementation and is energy efficient for the mobiles, which do not need to listen to the channel all the time. Some other protocols, however, employ a dynamic frame structure, with the objective to utilize the bandwidth more efficiently.

- Performance: The capacity of the network and QoS depend critically on the performance of the protocol in terms of packet dropping rate, delay, throughput, and utilization.

A scrutiny of the above four aspects reveals a large design space for uplink access control protocols, despite that not many such protocols are proposed for nomadic computing environments. Nevertheless, five recently suggested protocols, designed based on radically different philosophies, are selected for an extensive performance comparison with our proposed protocol. The protocols chosen are:

- RAMA [2]: resource auction multiple access, a protocol that employs a collision avoidance approach;

- RMAV [7]: reservation-based multiple access with variable frame, a protocol with a dynamic frame with variable length, designed to achieve a short delay at light load and high throughput at high load;

- DRMA [14]: dynamic reservation multiple access, a protocol with a dynamic frame structure in which the portion of bandwidth designated for user requests

${ }^{1}$ We refer to such services as simply data services hereafter for simplicity. is dynamically adjusted, designed to maintain system stability at high load;

- D-TDMA/FR [14]: a traditional dynamic TDMA protocol with a static frame structure;

- D-TDMA/VR [8]: a dynamic TDMA protocol based on a channel-adaptive variable-throughput physical layer;

In general, these previous protocols attempt to accommodate more file data transmission requests, which do not impose constraints on data delay, by exploiting the silence gaps of the voice requests, which require bounded-delay packet transmission and hence, enjoy a higher transmission priority than data users in that reservation is allowed for the former but not the latter. However, while sophisticated slot assignment strategies with articulated frame structures are proposed in these methods, none of them considers the effect of burst channel errors on protocol performance, let alone the investigation of exploiting the error characteristics to enhance performance. Essentially, these previous protocols are designed and analyzed based on the assumption that packet transmission through the wireless channel is error-free. However, because the geographically scattered mobile users inevitably suffer from different degrees of fading and shadowing effects, indeed a common drawback of previous protocols is that they assume the underlying physical layer always delivers a constant throughput, and as such, they may not be able to effectively utilize the precious bandwidth when the channel condition is swiftly varying among different users.

Our proposed uplink access control protocol, called CHARISMA (들 ISochronous Multiple Access), which is based on a channel adaptive physical layer. As will be detailed in Section III, CHARISMA is a dynamic TDMA (DTDMA) based protocol with one major distinctive feature: the user contention requests are gathered by the base-station in the first phase of the time frame without immediately announcing the information slots assignment right after each contention mini-slot. After all requests are received, the information slots are assigned to the users based on their respective CSI ranking. Performance gain is derived by supplying an additional input to the protocol, namely the channel state information (CSI) from the physical layer.

We have implemented all the six protocols on a common simulation platform, from which extensive performance results are obtained. The protocols are evaluated for test cases with and without request queues, which store transmission requests that survive the contention but fail to get assigned information slots. Three performance metrics, namely voice packet dropping rate, data delay, and data throughput, are considered. The balance of this paper is as 
follows. In the next section, we describe the models we used. In Section III, we describe in detail the functionality and characteristics of the proposed CHARISMA protocol. Section IV contains the performance results and our interpretations. The final section provides some concluding remarks.

\section{MODELS}

The cellular mobile information system considered in this paper, with a star topology as shown in Figure 1 (i.e., a cell phone network with file data transmission support), is aimed to support integrated voice and data services. As such, we assume that there are only two types of mobile requests, namely the voice requests and the data requests in the system. Voice packets are assumed to be delay sensitive while data packets are assumed to be delay insensitive. Thus, voice packets are labeled with deadlines. A voice packet will be dropped by the mobile device if the deadline expires before being transmitted. Such packet dropping has to be controlled to within a certain limit (e.g., below $1 \%$ as indicated in [6]) in order that the quality of service to the voice users is still acceptable. The source and contention models are summarized below.

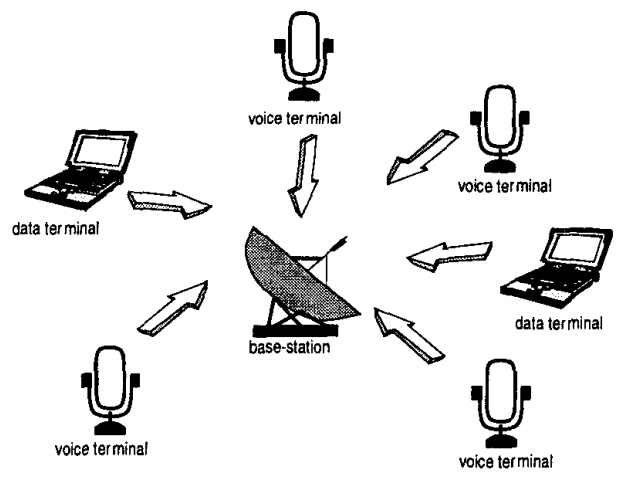

Fig. 1. System model for the uplink access of a wireless system with voice and file data requests.

- Voice Source Model: The voice source is assumed to be continuously toggling between the talkspurt and silence states. The duration of a talkspurt and a silence period are assumed to be exponentially distributed with means $t_{t}$ and $t_{s}$ seconds, respectively (as indicated by the empirical study in [6], $t_{t}=1$, and $t_{s}=1.35$ ). We assume a talkspurt and a silence period start only at a frame boundary.

- Data Source Model: The arrival time of file data generated by a mobile device is assumed to be exponentially distributed with mean equal to one second. The data size, in terms of number of packets, is also assumed to be exponentially distributed with mean equal to 100 packets. Again we assume that the packets arrive at a frame boundary.
- Request Contention Model: As in most previous studies, to avoid excessive collisions, even if a voice or data request has some packets awaiting to be sent, the mobile device will attempt to send a request at a request mini-slot only with a certain permission probability. The permission probability for submitting voice and data requests are denoted by $p_{v}$ and $p_{d}$, respectively.

A mobile device entering a new voice talkspurt or generating a new stream of data packets transmits an appropriate request packet in one of the request slots of the next frame. If there are more than one packet transmitted in the same request slot, collision occurs and none of the requests will be correctly received (we ignore the capture effect [1] in this paper). At the end of each request slot, the successful or unsuccessful request will be identified and broadcast by the base station. Due to the short propagation delay in a cellular network, the mobile devices can immediately know the request result. An unsuccessful mobile device can retry in the next request slot. On the other hand, a successful mobile device then transmits his/her information packet in the corresponding information slot in the current frame.

\section{The Proposed CHARISMA Protocol}

The CHARISMA ( $\underline{\mathrm{CH}}$ annel Adaptive Reservationbased ISochronous Multiple Access) is based on a novel concept-exploiting the synergy between two protocol layers instead of strictly following the traditional information hiding protocol design paradigm. Figure 2 highlights the differences in the designs among D-TDMA/FR, D-TDMA/VR, and CHARISMA.

\section{A. Structures of TDMA Frames}

In the uplink, a frame is divided into three subframes, namely, the request subframe, information subframe, and the pilot symbol subframe. Specifically, there are $N_{r}$ mini-slots in the request subframe for voice requests reservation and data requests contention. Again, a data request is not allowed to make reservation in the sense that even if a data request successfully seizes an opportunity to transmit in the current frame, he/she has to contend again in the next frame if he/she has some more data to send. There are $N_{i}$ information slots in the information subframe for the transmission of voice or data packets. Finally, there are $N_{b}$ slots in the pilot symbol subframe. On the other hand, a downlink frame is similarly partitioned into four subframes, namely the acknowledgment subframe, poll-for-CSI subframe, information subframe, and announcement subframe. The number of slots in the subframes are also given by $N_{r}, N_{b}, N_{i}$, and $N_{b}$, respectively. The detailed 


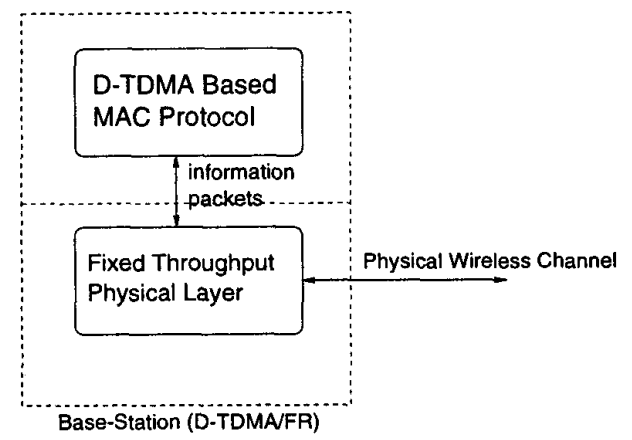

(a) D-TDMA/FR (fixed-throughput channel encoder)

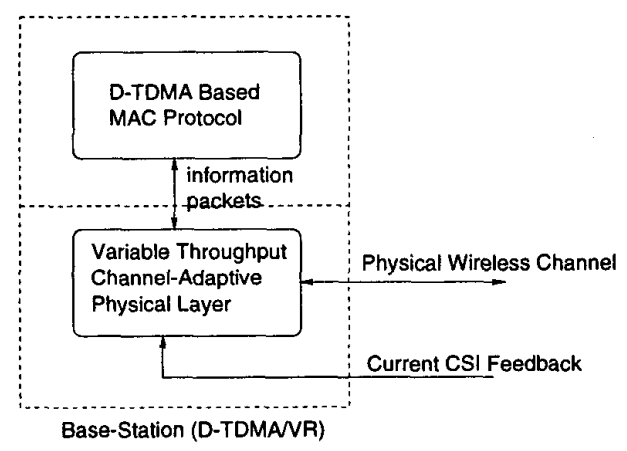

(b) D-TDMA/VR (adaptive channel encoder)

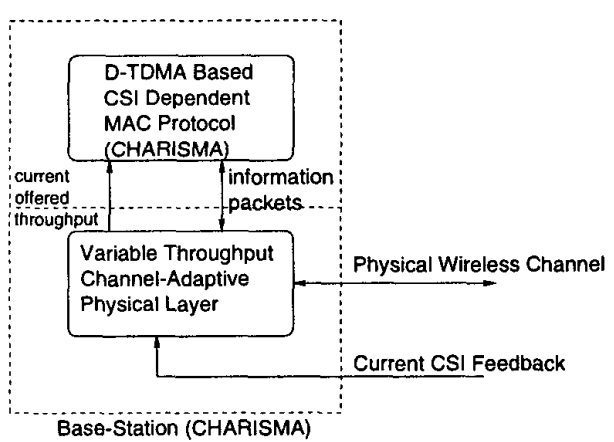

(c) CHARISMA (adaptive channel encoder with access control interaction)

Fig. 2. Conceptual models of the physical and access control layers. functions of each subframe will be elaborated later in Section III-C. The frame duration is $2.5 \mathrm{msec}$. Such a short frame duration has the advantage of shorter delay and is practicable in wideband systems [16].

\section{B. Variable Throughput Channel-Adaptive Physical Layer}

The CHARISMA protocol is designed based on a novel channel-state conscious concept such that the burst-error property of the radio channel is exploited to further enhance the multiple access system performance. Specifically, the wireless link between a mobile device and the base-station is characterized by two components, namely the fast fading component and the long-term shadowing component. Fast fading is caused by the superposition of multipath components and is therefore fluctuating in a very fast manner (on the order of a few msec). Long-term shadowing is caused by terrain configuration or obstacles and is fluctuating only in a relatively much slower manner (on the order of one to two seconds).

Let $c(t)$ be the combined channel fading which is given by:

$$
c(t)=c_{l}(t) c_{s}(t)
$$

where $c_{l}(t)$ and $c_{s}(t)$ are the long-term and shortterm fading components, respectively. Both $c_{s}(t)$ and $c_{l}(t)$ are random processes with a coherent time on the order of a few milli-seconds and seconds, respectively.

As usual, redundancy is incorporated to the information packet for error protection. To exploit the time-varying nature of the wireless channel, a variable-throughput channel-adaptive physical layer is employed as illustrated in Figure 3. Channel state information (CSI), $c(t)$, which is estimated at the receiver, is fed back to the transmitter via a low-capacity feedback channel. Based on the CSI, the level of redundancy and the modulation constellation applied to the information packets are adjusted accordingly by choosing a suitable transmission mode. Thus, the instantaneous throughput is varied according to the instantaneous channel state. In our study, a 6-mode variable-throughput adaptive bit-interleaved trellis coded modulation scheme (ABICM) is employed [10]. Transmission modes with normalized throughput ${ }^{2}$ varying from $1 / 2$ to 5 are available depending on the channel condition.

We assume the coherent time of the short-term fading is around ten msec which is much longer than an information slot duration. Thus, the CSI remains approximately constant within a frame and it follows that the transmission mode for the whole frame is

${ }^{2}$ Normalized throughput refers to the number of information bits carried per modulation symbol. 


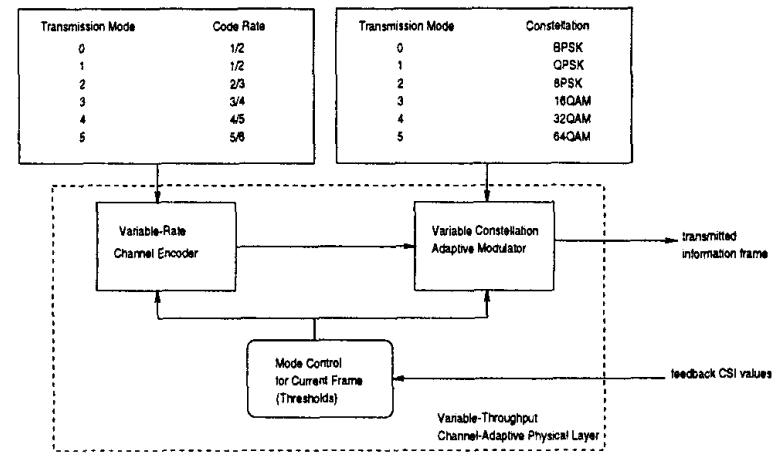

Fig. 3. A conceptual block diagram of the variable-throughput channel adaptive physical layer.

determined only by the current CSI level. Specifically, transmission mode $q$ is chosen if the feedback CSI, $\hat{c}$, falls within the adaptation thresholds, $\left(\zeta_{q-1}, \zeta_{q}\right)$. Here, the operation and the performance of the ABICM scheme is determined by the set of adaptation thresholds $\left\{\zeta_{0}, \zeta_{1}, \ldots\right\}$. In this paper, we assume that the ABICM scheme is operated in the constant BER mode [10]. That is, the adaptation thresholds are set optimally to maintain a target transmission error level over a range of CSI values. When the channel condition is good, a higher mode could be used and the system enjoys a higher throughput. On the other hand, when the channel condition is bad, a lower mode is used to maintain the target error level at the expense of a lower transmission throughput. Note that when the channel state is very bad, the adaptation range of the ABICM scheme can be exceeded such that the throughput (mode-0) becomes so low, making it impossible to maintain the targeted BER level. Given the above considerations about the channel state, the instantaneous throughput offered to the access control layer, denoted by $\rho$, is also variable and is therefore a function of the CSI, $c(t)$, and the target BER, $P_{b}$, denoted by $\rho=f_{\rho}\left(c(t), P_{b}\right)$.

\section{Multiple Access Control}

The operation of the CHARISMA protocol is divided into two phases, namely the request phase and transmission phase. In the request phase, mobile devices which have voice or data packets to transmit will send a request packet in one of the request slots, governed by the respective permission probability. The request packet is very short, occupying only a mini-slot. It contains the mobile device ID, request type (voice or data), packet deadline, number of information packets desired to transmit as well as some pilot symbols. If more than one mobile device send request packets in the same request slot, collision occurs and none of the request packets can be successfully received (i.e., capture effect is not considered in this study). After each request slot, an acknowledgment packet will be broadcast from the base-station through the acknowledgment slot in the downlink frame. The acknowledgment packet contains only the successful request packet ID. Mobile devices that fail to receive an acknowledgment will retransmit the request packet in the next request slot, again governed by the permission probability. On the other hand, successfully acknowledged devices will wait for announcement on the allocation schedule from the base-station.

Similar to traditional access control protocols, the number of request slots in the CHARISMA protocol, $N_{r}$, is slightly larger than the number of information slots, $N_{i}$, in order to provide more opportunities for users to contend for information slots. In the CHARISMA protocol, the $N_{r}$ mini-slots are used for gathering more mobile device requests as candidates for information time slot allocation. Specifically, the base-station first collects all requests in the current request phase as well as the backlog requests from the previous frames. All the requests will be assigned priorities which are computed according to the deadline, CSI, service type (voice or data), as well as the waiting time of the request (i.e., the number of elapsed frames since the request is acknowledged). Since the physical layer offers a variable throughput which is dependent on the CSI, the rationale behind the CHARISMA protocol is to give higher priority to the mobile devices that are in better channel condition in the bandwidth allocation process. The motivation of this strategy is that a user with better channel condition, with the support of the variable-throughput channel encoder, can enjoy a larger throughput and therefore, can use the system bandwidth more effectively. Nevertheless, for fairness's sake, information slots should also be allocated to mobile devices that are approaching their deadlines, despite their possibly worse channel states; otherwise, the queued information packets will be dropped. Thus, the requests are served in a prioritized manner according to their respective CSI, urgency, and data type (a voice request always gets a higher priority than a data request).

The CHARISMA protocol is reservation-based for voice requests only. As mentioned earlier, for a data request, even if information time slots have been assigned for its successfully acknowledged request, the allocation is meant only for the current frame and the user has to initiate another request if there are still some more data packets to be sent. By contrast, for a voice user, when information time slots have been assigned for its successfully acknowledged request, additional requests will be automatically generated by the base-station (hence, reservation) periodically at $20 \mathrm{msec}$ time intervals (the voice packet period). 
Thus, the voice user does not need to contend for request slots anymore for the whole remaining time in the current talkspurt. By avoiding unnecessary requests, the advantage of this reservation strategy is to reduce the contention collisions by the request slots.

\section{Request Queue}

All the above mentioned protocols, except $\mathrm{RMAV}^{3}$, can incorporate a request queue, which stores at the base-station the previous requests that survive the contention but are not allocated information slots. Such a request queue can further alleviate the capacity loss due to requests contention, especially when the traffic load is high. However, including a request queue inevitably increases the implementation complexity of the protocols. Thus, there is a trade-off. In our simulation study, we examine both the with and without queue versions of each protocol.

\section{Performance Results}

We have implemented the six protocols on a common simulation platform. In our experiments, we assume a transmission bandwidth of $320 \mathrm{KHz}$ for the TDMA frames. Bit rate of the speech source is $8 \mathrm{Kbps}$ which conforms to the values in GSM and CDMA systems. Due to space limitations, we include only the results for voice requests. The results of data requests indicate a similar trend and can be found in [9].

Because the quality of voice communication is determined by the average packet loss rate, we quantify the performance of voice requests by packet loss rate, $P_{\text {loss. }}$. Note that the packet loss probability is composed of two factors, namely, packet dropping and packet transmission error. On one hand, voice packet is delay sensitive and hence, voice packets are labeled with deadlines. A voice packet has to be discarded if its delay exceeds the deadline ${ }^{4}$. Such discarding constitutes the packet dropping at the mobile device. On the other hand, transmitted packet could be corrupted due to channel error and thus, packet transmission error results. The packet loss rate, $P_{\text {loss }}$, is given by:

$$
P_{\text {loss }}=\frac{N_{t x}-N_{r v}}{N_{t x}}
$$

where $N_{t x}$ and $N_{r v}$ are the number of transmitted voice packets and the number of voice packets received without error, respectively. The performance

\footnotetext{
${ }^{3}$ The RMAV protocol [7] inherently does not require a request queue because there is only one request contention opportunity in each frame so that there is only one winner.

${ }^{4}$ In this paper, the deadline of voice packet is assumed to be 20 msec after it is generated by the source.
}

of the six protocols in terms of packet loss rate versus the number of active voice user is shown in Figures 4 and 5 .

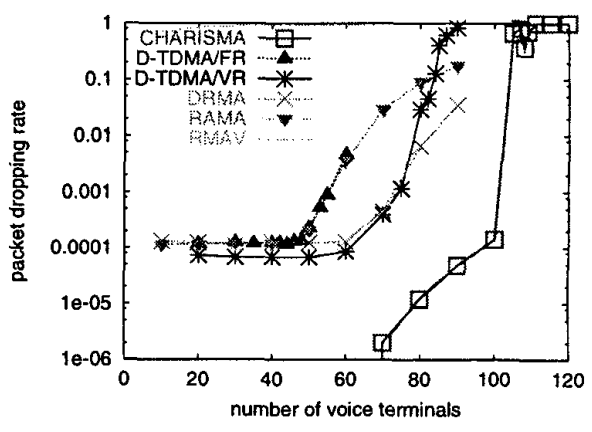

(a) without request queue

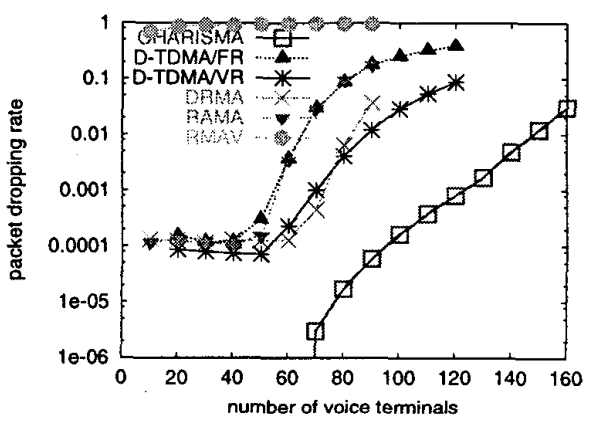

(b) with request queue

Fig. 4. Voice performance comparison (packet dropping rate vs. traffic load) with $N_{d}=0$.

A glance at Figures 4 and 5 clearly reveals that the CHARISMA protocol outperforms the other five protocols by a considerable margin in terms of voice packet dropping rate, while the relative rankings among the other fives are not very consistent in the six test scenarios. In addition, at low traffic low, the CHARISMA protocol almost incurs no packet loss but the other five protocols still have a certain level of packet loss. A close scrutiny reveals that such low load losses are due to transmission errors. Another general observation is that the RMAV protocol quickly becomes unstable even with a moderate number of voice users (e.g., 10). This demonstrates clearly that providing just one request contention opportunity can easily lead to instability. Specifically, consider the case without request queue and $N_{d}=0$ (see Figure $4(\mathrm{a})$ ). At the $1 \%$ voice packet dropping rate threshold, we can see that CHARISMA can accommodate approximately 100 


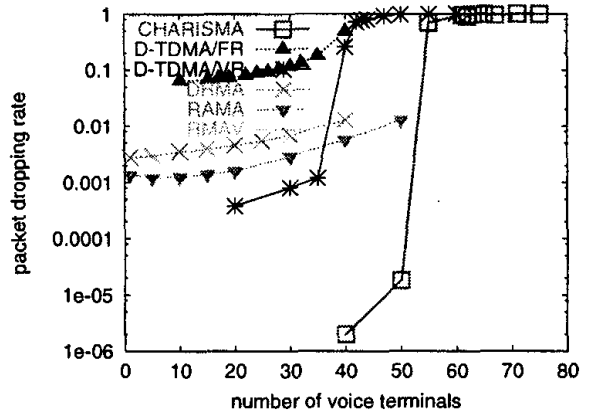

(a) without request queue

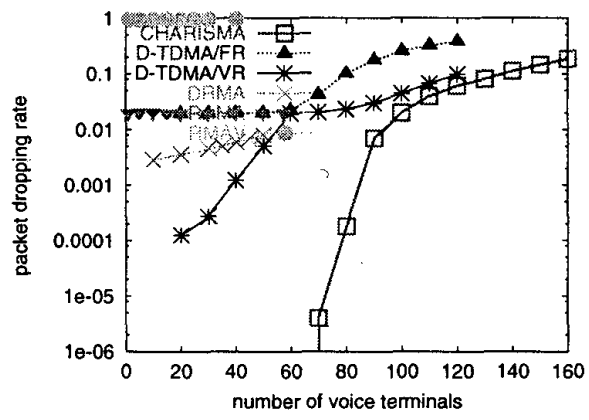

(b) with request queue

Fig. 5. Voice performance comparison (packet dropping rate vs. traffic load) with $N_{d}=20$.

voice users, while both DRMA and D-TDMA/VR can support only about 80 voice users (the former is slightly better than the latter in this case). Furthermore, the number of voice users supported by both RAMA and D-TDMA/FR is about 60. Thus, for this test scenario, the ranking of the six protocols is: CHARISMA, DRMA, D-TDMA/VR, RAMA, DTDMA/FR, RMAV. To summarize, the results without request queue indicate the following findings:

- With the intelligent CSI dependent scheduling, the CHARISMA protocol is able to utilize the bandwidth much more effectively. Indeed, because the scheduling process avoids allowing requests with poor channel states to get information slots, packet loss due to transmission errors is greatly reduced. Furthermore, at high load, the CHARISMA protocol can keep the packet dropping due to timeout at a low level.

- Despite that D-TDMA/VR also employs a variable-throughput physical layer, it does not outperform DRMA and RAMA by a great margin (i.e., those with fixed-throughput physical layer). Indeed, by examining the simulation traces, we find that the major benefit of using a variable-throughput physical layer in D-TDMA/VR is that packet loss due to transmission errors is reduced due to the added protection. Thus, it appears that exploiting the synergistic effect between the access control and physical layer (i.e., the CSI dependent scheduling in CHARISMA protocol) is much more important than using the variable-throughput physical layer alone.

- The RAMA protocol, with its collision avoidance property, exhibits a much more graceful performance degradation compared with CHARISMA, D-TDMA/VR, D-TDMA/FR, and RMAV, when the traffic load becomes very high. Similarly, the DRMA protocol also does not become unstable when system load is high due to its dynamic frame structure, which simply does not allow users to make requests at high load.

- When there are data users in the system, the request contention load becomes much higher because a data user repeatedly transmits requests until information slots are granted, as governed by the data permission probability $p_{d}$. Furthermore, as a data burst may consist of a few tens of packets, a data user that successfully gets some information slots may also repeat transmitting requests because reservation is not allowed for data users. Thus, every protocol accommodates less voice users when there are data users in the system.

Next we consider the performance of protocols with request queues. Comparing with those without request queues, the capacity of the CHARISMA and D-TDMA/VR protocols increase significantly. For example, consider the case with $N_{d}=0$, the CHARISMA protocol can accommodate about 160 voice users at the $1 \%$ packet dropping rate threshold, while for the case without request queue, only 100 voice users can be supported. The capacity of D-TDMA/VR also increases by about $25 \%$. For the CHARISMA protocol, incorporating a request queue can further increase the selection diversity in the CSI dependent scheduling process and, thus, the channel bandwidth can be utilized even more effectively. On the other hand, the request queue in the D-TDMA/VR protocol helps in reducing the packet losses due to severe requests contention.

A very interesting observation is that adding a request queue improves the performance of DRMA and RAMA only slightly. For the DRMA protocol, such an unexpected phenomenon can be explained as follows: the request queue in DRMA usually only contains a few pending requests because an inherent property of DRMA is that users are allowed to participate in requests contention when there are unused information slots. However, existence of unused information slots implies that successful requests will very likely be assigned information slots in the current frame, and thus, will not be queued. Thus, 
using a request queue does not help much. Put it in another way, the DRMA protocol inherently exhibits a "distributed requests queueing" propertyuser requests are "queued" at the user side (i.e., distributed) when there are no empty information slots. On the other hand, for the RAMA protocol, due to its collision avoidance property, all the auction slots will result in successful requests and the queued requests, if any, will very unlikely to have chances of getting services, especially when the traffic load is high. In summary, the fundamental function of a request queue is to alleviate the request contention and, as such, it is not useful for protocols with inherent stablizing property such as DRMA and RAMA. Finally, the ranking of protocols with request queue is: CHARISMA, D-TDMA/VR, DRMA, RAMA, DTDMA/FR, RMAV.

\section{CONClusions}

We have presented a new channel-adaptive uplink access control protocol for a mobile computing system. The proposed protocol, called CHARISMA, employs a variable-throughput adaptive channel encoder and modulator in the physical layer. A performance comparison of CHARISMA and five recently proposed related protocols, namely D-TDMA/VR, D-TDMA/FR, DRMA, RAMA, and RMAV is also described. These protocols are carefully selected such that they are devised based on rather orthogonal designed philosophies. The protocols are evaluated for test cases with and without request queues, which store transmission requests that survive the contention but fail to get assigned information slots. Our simulation results reveals that using the knowledge of the channel condition reported to the access control layer by the physical layer (in the CHARISMA protocol) is indeed a very useful component in achieving even higher performance in a wireless communication system where burst errors are the norm rather than exception.

\section{ACKNOWLEDGMENTS}

The authors would like to thank Professor Victor $\mathrm{Li}$ for his initial inspiration on the idea of joint design of protocol layers. This research was jointly supported by research initiation grants from the HKU CRCG under contract numbers 10202523 and 10202518, and by a grant from the Hong Kong Research Grants Council under contract number HKU7124/99E.

\section{REFERENCES}

[1] J. C. Ambak and W. van Blitterswijk, "Capacity of Slotted ALOHA in Raleigh Fading Channels," IEEE Journal on Selected Areas in Communications, vol. SAC-5, no. 2, pp. 261-268, Feb. 1987
[2] N. Amitay and L. J. Greenstein, "Resource Auction Multiple Access (RAMA) in the Cellular Environment," IEEE Transactions on Vehicular Technology, vol. 43, no. 4, pp. 1101-1111, Nov. 1994

[3] D. Barbara, "Mobile Computing and Databases--A Survey," IEEE Transactions on Knowledge and Data Engineering, vol. 11, no. 1, pp. 108-117, Jan./Feb. 1999.

[4] A. Datta, D. E. VanderMeer, A. Celik, and V. Kumar, "Broadcast Protocols to Support Efficient Retrieval from Databases by Mobile Users,". ACM Transactions on Database Systems, vol. 24, no. 1, pp. 1-79, Mar. 1999.

[5] R. Dube, C. D. Rais, and S. K. Tripathi, "Improving NFS Performance Over Wireless Links," IEEE Transactions on Computers, vol. 46, no. 3, pp. 290-298, Mar. 1997.

[6] J. Gruber and L. Strawczynski, "Subjective Effects of Variable Delay and Speech Clipping in Dynamically Managed Voice Systems," IEEE Transactions on Communications, vol. COM-33, vol. 8, pp. 801-808, Aug. 1985.

[7] D. G. Jeong, C.-H. Choi, and W. S. Jeon, "Design and Performance Evaluation of a New Medium Access Control Protocol for Local Wireless Data Communications," IEEE/ACM Transactions on Networking, vol. 3 , no. 6 , pp. $742-752$, Dec. 1995.

[8] M. Kawagishi, S. Sampei, and N. Morinaga, "A Novel Reservation TDMA-Based Multiple Access Scheme Using Adaptive Modulation for Multimedia. Wireless Communication Systems," Proceedings of VTC'98, pp. 112-116, 1998.

[9] Y.-K. Kwok and V. K, N. Lau, "A Novel ChannelAdaptive Uplink Access Control Protocol for Nomadic Computing," Technical Report, Department of Electrical and Electronic Engineering, HKU, January 2000.

[10] V. K. N. Lau, "Performance of Variable Rate BitInterleaved Coding for High Bandwidth Efficiency," Proceedings of $V T C^{\prime} 2000$, Tokyo, May 2000, accepted for publication and to appear.

[11] K. K. Leung, "An Update Algorithm for Replicated Signaling Databases in Wireless and Advanced Intelligent Networks," IEEE Transactions on Computers, vol. 46, no. 3, pp. 362-367, Mar. 1997.

[12] M. S. Mazer and C. L. Brooks, "Writing the Web While Disconnected," IEEE Personal Communications, pp. 3541 , Oct. 1998 .

[13] N. Mirghafori and A. Fontaine, "A Design for File Access in a Mobile Environment," Proceedings of the Workshop on Mobile Computing Systems and Applications, pp. 57$62,1995$.

[14] X. Qju and V. O. K. Li, "Dynamic Reservation Multiple (DRMA): A New Multiple Access Scheme for Personal Communication System (PCS)," ACM/Baltzer Wireless Networks, vol. 2, pp. 117-128, 1996 .

15] M. Satyanarayanan, "Mobile Information Access," IEEE Personal Communications, pp. 26-33, Feb. 1996.

[16] A. Urie, M. Streeton, and C. Mourot, "An Advanced TDMA Mobile Access System for UMTS," Proceedings of the IEEE PIMRC'94, 1994.

[17] M. D. Yocoub, Foundations of Mobile Radio Engineering, McGraw Hill, 2nd edition, 1986. 